\title{
RELAXATION TO EQUILIBRIUM IN DIFFUSIVE-THERMAL MODELS WITH A STRONGLY VARYING DIFFUSION LENGTH-SCALE*
}

\author{
PAUL CLAVIN $^{\dagger}$, LAURENT MASSE $^{\ddagger}$, AND JEAN-MICHEL ROQUEJOFFRE ${ }^{\S}$
}

\begin{abstract}
We consider reaction-diffusion equations with a strongly varying diffusion lengthscale. We provide a mathematical study of the relaxation towards the steady planar solution, in the context of infinitesimal disturbances whose wavelength is much shorter than the total thickness of the wave. The models under study are relevant in the description of ablation fronts encountered in inertial confinment fusion, when hydrodynamical effects are neglected.
\end{abstract}

Key words. Ablation front, relaxation, strongly varying diffusivity.

AMS subject classifications. 34E10, 34E20, 76R50.

\section{Introduction}

The paper is motivated by the the short wavelength stabilization of the wrinkled ablation front encountered in inertial confinement fusion (ICF). This will be shown to be in contrast with another thermal wave: the flame propagating in premixed gases; see [6] and [10]. The formulation of the problem is similar but the increase in temperature and the regime of hydrodynamical instability are different. The main characteristics of ablation fronts in ICF may be summarized as follows. Firstly, owing to a large variation of heat conductivity, the diffusion length-scale varies strongly across the unperturbed planar wave. Secondly, the Rayleigh-Taylor instability is such that the physically relevant disturbances have a wavelength that lies in an intermediate range, between the shortest length-scale and the largest one (total thickness of the wave). By comparison, the diffusion length-scale in flames does not vary much across the flame structure and, in addition, the relevant disturbances have a wavelength which is not shorter than the total thickness of the wave [5]. As suggested in [6], the difference in the behaviours is enlightened by the study of a much simpler problem, namely the relaxation of wrinkled reaction-diffusion waves, free from hydrodynamical effects. This is precisely the purpose of the present paper.

1.1. Diffusive-thermal models. We are interested in the relaxation rate of the following two-dimensional diffusive-thermal model, written in non-dimensional form:

$$
\left\{\begin{aligned}
\left(\frac{1}{T}\right)^{n} T_{t}-\operatorname{div}(\lambda(T) \nabla T)+c T_{x} & =F(T) \quad(t>0),(x, y) \in \mathbb{R} \times \mathbb{R}, \\
T(t,-\infty, y) & =\varepsilon^{\prime}, \quad T(t,+\infty, y)=1, \\
T\left(t, x, y+\frac{2 \pi}{k}\right) & =T(t, x, y) .
\end{aligned}\right.
$$

Here $T$ is the reduced temperature scaled by its maximum value at the hot side, $0 \leq \varepsilon^{\prime}<1 . F(T)$ is the nonlinear term describing the heat release rate, $F\left(\varepsilon^{\prime}\right)=F(1)=$

\footnotetext{
${ }^{*}$ Received: October 8, 2008; accepted (in revised version): May 16, 2010. Communicated by Jack Xin.

†IRPHE (UMR CNRS 6594); Technopôle de Château-Gombert, 49, rue Joliot Curie, (Paul. Clavin@irphe.univ-mrs.fr).

${ }^{\ddagger}$ CEA Bruyères le Châtel, Ile de France, France (laurent.masse@cea.fr).

$\S$ Institut de Mathématiques (UMR CNRS 5219), Université Paul Sabatier, 118 route de Narbonne, 31062 Toulouse Cedex, France (jean-michel.roquejoffre@math.univ-toulouse.fr).
} 
$0, F(T)>0$ on $] \varepsilon^{\prime}, 1[$, and $c$ is the constant propagation velocity of the unperturbed planar wave. This quantity is an eigenvalue value of the steady version of (1.1), $T_{t}=0$. Finally, the $\frac{2 \pi}{k}$-periodicity in $y$ (with $k>0$ very large) accounts for the initial wrinkling of the front - that we may define as the region where the temperature is equal to $2 \varepsilon^{\prime}$.

We take a reduced heat conductivity of the form

$$
\lambda(T)=T^{m-1}, \quad m>2 .
$$

According to the elementary kinetic theory of gases, one has $m \approx 3 / 2$ in flames, and the temperature ratio $1 / \varepsilon^{\prime}$ is about 6 to 9 . For ablation fronts in ICF the temperature ratio is much higher, $1 / \varepsilon^{\prime} \approx 50$, and, according to the Spitzer law for the conduction by the electrons of a dilute plasma, the power index is $m=7 / 2$. In any case we will take $m>2$.

We consider small values of $\varepsilon^{\prime}$, so that we are dealing with a conduction lengthscale $\lambda(T) / c$ that varies strongly across the wave from $\varepsilon^{\prime m-1} / c$ to $1 / c$. In view of the comparison with the results in [6] and [10], it is worth stressing that, according to the scaling of the reduced temperature $T$ in (1.1), the length-scale in (1.1) is reduced by the conduction length-scale at the hot side.

For reasons discussed below, we consider two values of the exponent, $n=0$ and $n=1$.

1.2. Physical content of the models. Equation (1.1) with $n=0$ corresponds to the usual diffusive-thermal model, written in the referential frame of the unperturbed planar wave. It is obtained from the equation for the conservation of energy in a low Mach number flow of perfect gas with a unity Lewis number, when the variation of density, $\rho=1 / T$, and the flow modification are omitted. For disturbances with a wavelength larger than the total thickness of the thermal wave, the relaxation rate is proportional to the square of the wave number, as it is expected from the diffusion equation. For such disturbances, the density induced hydrodynamical effects do not change the relaxation rate qualitatively; they modify only the coefficient of proportionality [5].

The situation is different for very short wavelengths, since the form of the relaxation law is modified by the density variation. In order to point out this effect we introduce the model (1.1) with $n=1$, so that the variation of density is taken into account only in the inertial term. This artificial model is not Galilean invariant. Obviously, this is not the case for the basic equations describing the conservation of mass, momentum, and energy, when the full hydrodynamic effects are included properly. A simple Galilean invariant model may be obtained by neglecting only the transverse flow. In such an intermediate model, the quantity $c$ in (1.1) (with $n=1$ ) is the mass flux, $c=w / T$, which is a function of $x, y$ and $t$, and the continuity equation

$$
(1 / T)_{t}+(w / T)_{x}=0
$$

should be added. However the simpler model constituted by the single Equation (1.1) with $n=1$ and $c$ constant is worth investigating. First, it keeps the basic onedimensional wave (see Section 2) as a stationary solution (we could think of replacing the $c T_{x}$ term by $\frac{c T_{x}}{T}$, but this would break the physical wave structure that we wish to keep). Second, we show in this paper that it describes a relaxation rate at small wavelengths (see Theorem 1.1 below) which is indeed close to the previous 
approximate results obtained within the framework of the basic model taking fully into account the hydrodynamic effects; see [10] and the references herein. This strongly suggests that the dominant mechanism of relaxation is included in model (1.1) with $n=1$. A study of the more complete model will be undertaken somewhere else.

1.3. The temperature cutoff and the linear equation. It is customary [3] to shift the unknown $T$ to

$$
\lambda=T^{m-1},
$$

the function $\lambda$ is - at least in the porous media equation - Lipschitz in the vicinity of its zero set [3], and smooth up to the boundary of its zero set [4]. It satisfies

$$
\left\{\begin{aligned}
\frac{\lambda_{t}}{\lambda^{\frac{n}{m-1}}}-\lambda \Delta \lambda+c \lambda_{x} & =f(\lambda)+\frac{1}{m-1}|\nabla \lambda|^{2} \quad(t>0),(x, y) \in \mathbb{R} \times \mathbb{R}, \\
\lambda(t,-\infty, y) & =\varepsilon, \quad \lambda(t,+\infty, y)=1 \\
\lambda\left(t, x, y+\frac{2 \pi}{k}\right) & =\lambda(t, x, y)
\end{aligned}\right.
$$

where we have written

$$
\varepsilon=\varepsilon^{\prime m-1} \text { and } f(\lambda):=(m-1) u^{\frac{m}{m-1}} F\left(\lambda^{\frac{1}{m-1}}\right) .
$$

From now on we assume the existence of a cutoff $\theta \in] 0,1[$ such that

$$
f \equiv 0 \quad \text { on } \quad[0, \theta], \quad \text { and } \quad f>0 \quad \text { on }] \theta, 1[,
$$

Moreover $f$ is smooth and is such that Problem (1.4) has a unique steady solution denoted $p(x)$, with $c$ of order unity for $m>1$ and $\varepsilon \in] 0,1[$. It is easy to show that the velocity $c(\varepsilon)$ goes to a positive constant as $\varepsilon \rightarrow 0$; see the next section.

The steady planar wave $p(x)$ is stable, and the goal of the paper is to compute the relaxation rate of a solution to (1.4), for small $\varepsilon$, and for a highly wrinkled initial disturbance; see below the last sentence of this section. Consider the linearisation of (1.4) around $p, \lambda=p(x)+u(t, x, y)$,

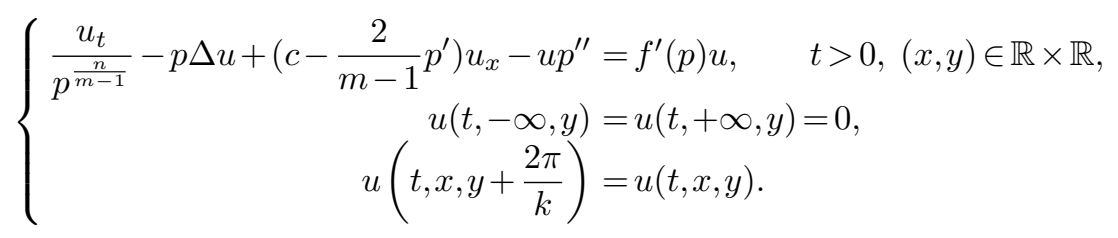

Introduce, as is usual, the ansatz

$$
u(t, x, y)=e^{-s t+i k y} v(x) ;
$$

where the wrinkling of the front is accounted for by the exponential of $i k y$. The equation for $v$ becomes

$$
-p v^{\prime \prime}+\left(c-\frac{2}{m-1} p^{\prime}\right) v^{\prime}-p^{\prime \prime} v=\left(\frac{s}{p^{\frac{n}{m-1}}}-k^{2} p+f^{\prime}(p)\right) v, \quad v( \pm \infty)=0 .
$$

The two characteristic exponents of $(1.7)$ at $x \rightarrow-\infty(p \rightarrow \varepsilon, f=0)$ are

$$
r_{ \pm}=\frac{c \pm \sqrt{c^{2}+4 \varepsilon\left(\varepsilon k^{2}-s \varepsilon^{-\frac{n}{m-1}}\right)}}{2 \varepsilon} .
$$


From now on, the modulus of the wave vector $k$ will be denoted by $k$ for simplicity. We are interested in the range of intermediate wavelengths $1 \ll k \ll \varepsilon^{-1}$. The inequality $1 \ll k$ insures that the wavelength is much shorter than the total thickness of the wave. If we anticipate that

$$
s>0 \text { and } 0<\varepsilon\left(s \varepsilon^{-\frac{n}{m-1}}-\varepsilon k^{2}\right) \ll 1,
$$

we have $0<r_{-}<r_{+}$with $r_{+} \sim c / \varepsilon$. The condition assumed in the whole paper will be

$$
1 \ll k \ll k^{2} \ll \varepsilon^{-1} \text {. }
$$

1.4. Contents. We consider here the limit (1.9), and look for positive solutions of (1.7) of maximal decay, i.e. those decaying at $-\infty$ like $e^{r_{+} x}$ with $r_{+} \sim c / \varepsilon$. The corresponding eigenvalue is then called the principal eigenvalue, and on the whole line the problem of its existence can be quite intricate; see [2], for instance. Here there is only one, called $s$, and our main result elucidates its behaviour in the limit $\varepsilon \ll k^{-1}$. The case $n=0$ was analysed for the first time, in a formal fashion, in [6]. For asymptotics in a different (semi-classical) regime, see [8]. Here is our main result:

Theorem 1.1. Choose $m>2$. For $n=1$ there is $\nu_{m}>0$, independent of $\varepsilon$ and $k$, such that, in the limit (1.9) we have:

$$
s \sim \nu_{m} \frac{k^{\frac{m-2}{m-1}}}{\ln (1 / k \varepsilon)} \text { if } n=1, \quad s \sim c k \text { if } n=0 .
$$

It may at first sight look restrictive to consider solutions of (1.7) having maximal decay. We claim that it is a physically natural choice; let us indeed recall that the conductivity $\lambda$ in (1.4) is of the order $\varepsilon$ and that — as will be seen in Section 2 below - its derivative (or the deviation from its limit at $-\infty$ ) decays like $e^{c x / \varepsilon}$ as $x \rightarrow-\infty$. In the limit $\varepsilon \rightarrow 0$, both quantities would be 0 and the limiting problem for (1.4) would be a free boundary problem in which the conductivity would be 0 ahead of the front. A perturbation of the front has to respect this setting, and solutions of (1.7) with minimal decay certainly do not respect this. This is why we are discarding them.

One may also wonder whether an eigenvalue would translate into an actual relaxation rate to the basic solution. We will explain why this is so in Section 5 below.

The presentation starts with a brief Section 2 concerning the steady solution. In Section 3, we prove a general result stating that models like (1.7) actually have at most one principal eigenvalue; we could have done it directly on our model, but we think that the result is of independent interest. The main bulk of the proof of Theorem 1.1 is given in Section 4; assuming that a positive solution to (1.5) exists, we prove the claimed asymptotics. In Section 5, the results are discussed and compared with the previous approximate results obtained when the hydrodynamical effects are included.

\section{The steady solution}

Plugging the ansatz $\lambda(t, x, y)=p(x)$ into (1.4) yields the governing equation of the planar wave propagating at constant velocity $c$,

$$
-p p^{\prime \prime}+\left(c-\frac{1}{m-1} p^{\prime}\right) p^{\prime}=f(p), \quad p(-\infty)=\varepsilon, p(+\infty)=1 .
$$

To eliminate the translational invariance we use the additional condition $p(x=0)=\theta$ in this section. Here is the relevant information on (2.1): 
Proposition 2.1. Problem (2.1) has a unique solution $\left(c_{\varepsilon}, p_{\varepsilon}\right)$. The sequence $\left(c_{\varepsilon}\right)_{\varepsilon>0}$ and the sequence of profiles $\left(p_{\varepsilon}\right)_{\varepsilon>0}$ converge respectively, as $\varepsilon \rightarrow 0$, to a unique $c_{0}>0$ and a unique profile $p_{0}(x)$ satisfying,

$$
\begin{aligned}
x<-\frac{\theta}{(m-1) c_{o}}: & p_{0}(x)=0 \\
-\frac{\theta}{(m-1) c_{o}} \leq x \leq 0: \quad & p_{0}(x)=\theta+(m-1) c_{o} x \\
x>0: & \left\{\begin{array}{c}
-p_{0} p_{0}^{\prime \prime}+\left(c_{0}-\frac{p_{0}^{\prime}}{m-1}\right) p_{0}^{\prime}=f\left(p_{0}\right), \\
p_{0}(0)=\theta, p_{0}^{\prime}(0)=(m-1) c_{0}, p_{0}(+\infty)=1 .
\end{array}\right.
\end{aligned}
$$

The result is quite standard and its proof will only be sketched. We note that any solution of (2.1) is strictly increasing in $x$ (see [1] and Section 4.2) - the model is quite similar and the results apply word by word. Consequently, $\varepsilon<p_{\varepsilon}(x)<1$ for any solution $p_{\varepsilon}(x)$ of (2.1). We now delete the $\varepsilon$-dependence of $c_{\varepsilon}$ and $p_{\varepsilon}$ for simplicity.

1. $x<0$. Set $U(p(x))=p^{\prime}(x)$; this is licit since $p^{\prime}>0$. Then

$$
-p \frac{d U}{d p}+c-\frac{U}{m-1}=0, \quad U(\varepsilon)=0,
$$

yielding

$$
U(p)=\left(1-\left(\frac{\varepsilon}{p}\right)^{\frac{1}{m-1}}\right)(m-1) c
$$

and, in turn,

$$
p^{\prime}(0)=\left(1-\left(\frac{\varepsilon}{\theta}\right)^{\frac{1}{m-1}}\right)(m-1) c .
$$

2. $x>0$. Consider, for any $\alpha>0$, the Cauchy problem

$$
\begin{aligned}
-p p^{\prime \prime}+\left(c-\frac{1}{m-1} p^{\prime}\right) p^{\prime} & =f(p) \text { on } \mathbb{R}_{+}, \\
p(0)=\theta, p^{\prime}(0) & =\alpha,
\end{aligned}
$$

with the additional condition $p(+\infty)=1$. From [1] and Section 4.2, we may find $c_{*}>0$ and a decreasing function $c \in\left[0, c_{*}\left[\mapsto \alpha_{0}(c)\right.\right.$ such that the solution of $(2.7)$ satisfies $p(+\infty)=1$ if and only if $\alpha=\alpha_{0}(c)$. Moreover we have $\alpha_{0}\left(c_{*}^{-}\right)=0$. Using Step 1 and the continuity of $p^{\prime}$ at $x=0$ we infer that $p(x)$ satisfies (2.1) if and only if

$$
\left(1-\left(\frac{\varepsilon}{\theta}\right)^{\frac{1}{m-1}}\right)(m-1) c=\alpha_{0}(c) .
$$

This equation has a unique solution because $\alpha_{0}$ is decreasing. The convergence of the corresponding solution $c_{\varepsilon}$ to the unique solution of $\alpha_{0}(c)=(m-1) c$ follows.

Identities (2.2) and (2.3) follow directly from (2.5) as shown now. By setting

$$
z=\varepsilon^{-1}(m-1) c x, \quad \text { and } \quad q(z)=\varepsilon^{-1} p,
$$

we get

$$
d q / d z=1-q^{-\frac{1}{m-1}}, \quad q(-\infty)=1^{+} .
$$


This shows that $p(x)$, the solution to (2.1), presents a boundary layer of thickness $\varepsilon$ in which $p=O(\varepsilon)$ with $p^{\prime}$ varying from $0^{+}(z \rightarrow-\infty)$ to

$$
(m-1) c[1+o(1))] \quad(q \gg 1) .
$$

According to the boundary condition at the hot side $p(x=0)=\theta$, the outer solution reads

$$
q \gg 1: \quad p \sim(m-1) c x+\theta .
$$

From now on we choose the origin of the $x$-axis inside the boundary layer, $p(x=$ $0)=O(\varepsilon)$, in such a way that the outer solution (2.9) takes the simple form

$$
x \gg \varepsilon: \quad p \sim(m-1) c x, \quad q \sim z, \quad z=\varepsilon^{-1}(m-1) c x .
$$

\section{First eigenvalues of elliptic operators on the line}

In this section, we consider the general eigenvalue problem

$$
L u:=-u^{\prime \prime}+a(x) u^{\prime}+b(x) u=\lambda c(x) u \text { on } \mathbb{R}, \quad u( \pm \infty)=0,
$$

The coefficients $a$ and $b$ are bounded and smooth; moreover we assume that the following quantities $a_{ \pm}=\lim _{x \rightarrow \pm \infty} a(x), \quad b_{ \pm}=\lim _{x \rightarrow \pm \infty} b(x), \quad c_{ \pm}=\lim _{x \rightarrow \pm \infty} c(x)$ exist; we suppose also that

$$
a_{-}>0, \quad 0<c_{+} \leq c(x)<c_{-}, \quad \frac{c_{-}}{b_{-}} \leq \frac{b(x)}{c(x)} .
$$

These assumptions contain those on the coefficients of problem (1.7); indeed we have, with the notations of (3.1),

$$
a=\frac{1}{p}\left(c-\frac{2 p^{\prime}}{m-1}\right) ; \quad b=k^{2}-\frac{p^{\prime \prime}+f^{\prime}(p)}{p} ; \quad c=\frac{1}{p^{\frac{n+m-1}{m-1}}},
$$

and the last assumption of (3.2) is true because of the regime (1.9) of the parameters.

Theorem 3.1. Problem (3.1) has at most one unique principal eigenvalue $\lambda_{0}$; moreover the set of eigenfunctions is one-dimensional. If such a principal eigenvalue exists, we have $\frac{b_{-}}{c_{-}}<\lambda_{0} \leq \frac{b_{+}}{c_{+}}$.

The upper bound on $\lambda_{0}$ is exactly what we need to perform safely the inner expansions of the next section; it indeed yields, with the notations of that section, $s=O\left(k^{2}\right)$. We will not look for sufficient conditions for the principal eigenvalue to exist; our argument in the next section shows that it exists.

\section{Proof of Theorem 3.1.}

Proof. Assume that $\lambda_{0}$ exists; if $\lambda_{0}<\frac{b_{-}}{c_{-}}$, then $b-\lambda_{0} c$ is bounded away from 0 , and the maximum priciple ensures that $u \equiv 0-$ a contradiction. If $\lambda_{0}=\frac{b_{-}}{c_{-}}$, the maximal decay of $u$ at $-\infty$ and the fact that $b-\lambda c$ is bounded away from 0 for $x$ large enough still allow us to apply the maximum principle, and conclude that $u \equiv 0$. If $\lambda_{0}>\frac{b_{+}}{c_{+}}$, the characteristic exponents of (3.1) at $+\infty$ are positive; thus $u$ cannot 
decay at $+\infty$. This restricts therefore the range of $\lambda$. The one-dimensionality of the eigenspace follows easily: indeed, the characteristic exponents of (3.1) are

$$
r_{-}^{1}(\lambda):=\frac{a_{-}-\sqrt{a_{-}^{2}+4\left(b_{-}-\lambda c_{-}\right)}}{2}, r_{-}^{2}(\lambda):=\frac{a_{-}+\sqrt{a_{-}^{2}+4\left(b_{-}-\lambda c_{-}\right)}}{2},
$$

so that $r_{-}^{1}(\lambda)<0<r_{-}^{2}(\lambda)$ and the space of solutions with maximal decay is really one-dimensional.

Assume now that $\lambda_{1}<\lambda_{2}$ are two principal eigenvalues, with two solutions $u_{1}(x)$ and $u_{2}(x)$. Note that the strong maximum principle imposes $u_{i}>0$ on $\mathbb{R}$. We will prove the existence of $q_{0}>0$ such that $q_{0} u_{2} \geq u_{1}$, and such that there exists $x_{0} \in \mathbb{R}$ for which we have $q_{0} u_{2}\left(x_{0}\right)=u_{1}\left(x_{0}\right)$; the existence of this contact point contradicts the strong maximum principle, and thus proves the uniqueness of the principal eigenvalue. To prove the existence of $q_{0}$ and $x_{0}$, there are two steps: first, we prove that $q u_{2} \geq u_{1}$ for large $q$. Because of (3.2), there are $x_{+}>0$ and $\rho>0$ such that $b(x)-\lambda_{1} c(x) \geq \rho$ if $x \geq x_{+}$. On the interval $\left.]-\infty, x_{+}\right]$we claim that $q u_{2}-u_{1} \geq 0$. Indeed, the maximal decay assumption implies, by the strict ordering of the characteristic exponents, that

$$
u_{i}(x) \sim q_{i} e^{r_{2}^{+}\left(\lambda_{i}\right) x}
$$

with $q_{i}>0$, so, $q u_{2}-u_{1}>0$ for large negative $x$ as soon as $q>\frac{q_{1}}{q_{2}}$. Increasing $q$ by a large amount gives $q u_{2}-u_{1}>0$ on $\left.]-\infty, x_{+}\right]$. Finally, we have

$$
\left(L-\lambda_{1} c\right)\left(q u_{2}-u_{1}\right)=\left(\lambda_{2}-\lambda_{1}\right) u_{1} \geq 0,
$$

and the zero-order coefficient of $L-\lambda_{1} c$ is positive for $x \geq x_{+}$; this implies, by the maximum principle: $q u_{2}-u_{1} \geq 0$ everywhere.

We may now consider the smallest $q>0$ such that $q u_{2}-u_{1} \geq 0$; call it $q_{0}$. From the strong maximum principle, $q_{0} u_{2}-u_{1}>0$ everywhere, so by (3.4) there is $\delta_{0}>0$ such that $\left(q_{0}-\delta\right) u_{2}-u_{1} \geq 0$ for $\delta \leq \delta_{0}$ on $\left.]-\infty, x_{+}\right]$; this, by the same argument as above, propagates to the whole line and contradicts the minimality of $q_{0}$. Hence the existence of the contact point, but it contradicts the strong maximum principle, unless $u_{1} \equiv u_{2}$, but this implies then $\lambda_{1}=\lambda_{2}$.

\section{Asymptotic analysis of (1.7)}

We are going to prove the estimate for $s$ in Theorem 1.1 by matching an inner solution (boundary layer at the cold side) with an outer solution through an additional layer that must be taken into account.

Theorem 3.1 ensures that the sought for principal eigenvalue is less than $k^{2}$, which will always be assumed to be much smaller than $\varepsilon^{-1}$. Refined conditions in the course of the proof will show up; however this will allow us to treat, in the inner layer expansions - i.e. the subsection below - the terms containing $s$ as lower-order perturbations. However, this estimate will not be good enough in the outer expansions as we will have to assume it to be $o\left(k^{1-\frac{1}{m-1}}\right)$. This will be made precise in due course.

4.1. Boundary layer at the cold side (inner solution). We anticipate that the solution of (1.7) presents a boundary layer similar to the steady solution, and we investigate the corresponding inner solution in this section. For $a \in(0,1)$ to be chosen later on, let us normalise the solution $v$ of (1.7) in such a way that

$$
v\left(x_{\varepsilon}\right)=1, \quad x_{\varepsilon}=\varepsilon^{1-a} .
$$


The exponent $a$ will be chosen so that $x=x_{\varepsilon}$ corresponds to the border of the boundary layer - namely to an intermediate region suitable for matching the inner solution with the outer solution. Let us introduce the inner quantities $\xi$ and $q(\xi)$ :

$$
\xi=\varepsilon^{-1} x, \quad q(\xi)=\varepsilon^{-1} p(\varepsilon \xi), \quad \xi_{\varepsilon}=\varepsilon^{-1} x_{\varepsilon}=\varepsilon^{-a}, \quad \lim _{\varepsilon \rightarrow 0} \xi_{\varepsilon}=\infty .
$$

Denote by $L$ the differential operator

$$
L=-q \frac{d^{2}}{d \xi^{2}}+\left(c-\frac{2}{m-1} q^{\prime}\right) \frac{d}{d \xi}-q^{\prime \prime} .
$$

According to the preceding section, $L q^{\prime}=0$ with

$$
\xi \rightarrow-\infty: q-1=O\left(e^{c \xi}\right), \quad \lim _{\xi \rightarrow+\infty} q^{\prime}(\xi)=c(m-1) ;
$$

see (2.8) and (2.10). Looking for a solution of (1.7) with maximal decay and satisfying (4.1) leads to

$$
\left\{\begin{array}{rlrl}
L v & =\varepsilon\left(\frac{\varepsilon^{-\frac{n}{m-1}} s}{q^{\frac{n}{m-1}}}-\varepsilon k^{2} q\right) v, & & (\xi \in]-\infty, \xi_{\varepsilon}[), \\
v\left(\xi_{\varepsilon}\right) & =1, & & (\xi \rightarrow-\infty) . \\
v(\xi) & =O\left(e^{\rho+\xi}\right), &
\end{array}\right.
$$

Recall that $\rho_{+} \sim c$. The only solution to $L v_{0}=0$ with maximal decay, satisfying $v_{0}\left(\xi_{\varepsilon}\right)=1$, is $v_{0}=q^{\prime} / q^{\prime}\left(\xi_{\varepsilon}\right)$. According to $(4.4)$, one has $q^{\prime}\left(\xi_{\varepsilon}\right)=c(m-1)(1+o(1))$. The standard idea is to look for the solution of (4.5) in the limit (1.9), of the form

$$
v(\xi)=v_{0}(\xi)+\varepsilon v_{1}(\xi)+\varepsilon v_{2}(\xi), \quad v_{0}=\frac{q^{\prime}(\xi)}{c(m-1)}, \quad v_{2}=o\left(v_{1}\right),
$$

with

$$
L v_{1}=h, \quad h \equiv\left(\frac{\varepsilon^{-\frac{n}{m-1}} s}{q^{\frac{n}{m-1}}}-\varepsilon k^{2} q\right) v_{0}, \quad v_{1}\left(\xi_{\varepsilon}\right)=0, \quad \lim _{\xi \rightarrow-\infty} e^{-c \xi / 2} v_{1}(\xi)=0 .
$$

The last condition imposed on $v$ looks non-optimal; in fact it does imply the maximal decay due to the fact that the space of solutions decaying faster than $e^{c \xi / 2}$ is onedimensional. Looking for the solution to (4.7) in the form $v_{1}=q^{\prime} \Phi$ leads to

$$
\alpha \Phi^{\prime}-\Phi^{\prime \prime}=g, \quad g=\frac{h}{q q^{\prime}}, \quad \alpha=\frac{c(m-1)-2 q^{\prime}}{(m-1) q}-2 \frac{q^{\prime \prime}}{q^{\prime}},
$$

where $L q^{\prime}=0$ has been used. According to (4.4),

$$
\lim _{\xi \rightarrow-\infty} q^{\prime \prime} / q^{\prime}=-c, \quad \lim _{\xi \rightarrow-\infty} \alpha=-c, \quad \lim _{\xi \rightarrow-\infty} g=\frac{\left(\varepsilon^{-\frac{n}{m-1}} s-\varepsilon k^{2}\right)}{c(m-1)},
$$

and the only solution of (4.8) which does not blow up exponentially fast with $-\xi$ when $\xi \rightarrow-\infty$ is

$$
\Phi^{\prime}=-\int_{-\infty}^{\xi} g(\eta) \exp \left(\int_{\eta}^{\xi} a(\beta) d \beta\right) d \eta
$$


so that the solution to (4.7) is

$$
v_{1}(\xi)=q^{\prime}(\xi) \int_{\xi}^{\xi_{\varepsilon}} \int_{-\infty}^{\zeta} \exp \left(\int_{\eta}^{\zeta} \alpha(\beta) d \beta\right) \frac{h(\eta)}{q(\eta) q^{\prime}(\eta)} d \eta d \zeta .
$$

The above computation ensures that one can devise an expansion of $v$ accurate to the order $o(\varepsilon)$ in the boundary layer. The argument — only sketched here — is by now standard; see, for instance, [7]. Denote $w(\xi)=e^{-c \xi / 2}$ and

$$
\left.\left.\left.B_{w, 0}=\left\{v \in U C(]-\infty, \xi_{\varepsilon}\right]\right): \quad w v \in U C(]-\infty, \xi_{\varepsilon}\right]\right\} .
$$

Here $U C(I)$ is the space of all bounded, uniformly continuous functions on the interval $I$. We also denote by $B_{w, n}$ the subspace of all functions of $B_{w, 0}$ whose derivatives up to order $n$ are $B_{w, 0}$. Finally, $B_{w, 0}^{0}$ denotes the subspace of all functions of $B_{w, 0}$ vanishing at $\xi^{\varepsilon}$. According to (4.8), the operator $L$ is an isomorphism from $B_{w, 2}$ to $B_{w, 0}^{0}$ and its inverse norm is controlled by $\varepsilon^{-a}$. This ensures that

$$
v(\xi)=v_{0}(\xi)+\varepsilon v_{1}+O\left(\varepsilon^{2-a}\right), \quad v^{\prime}(\xi)=v_{0}^{\prime}(\xi)+\varepsilon v_{1}^{\prime}+O\left(\varepsilon^{2-a}\right):
$$

the desired result.

We may now compute the slope of the solution to (4.5) at the exit of the boundary layer toward the hot side, $d v /\left.d x\right|_{+}$, in the region where $x$ is close to $x_{\varepsilon}$, namely:

$$
\left(\xi-\xi_{\varepsilon}\right) / \xi_{\varepsilon}=o(1), \quad \xi_{\varepsilon}=\varepsilon^{-a} .
$$

According to (4.4), we have $\lim _{\xi \rightarrow+\infty} q^{\prime \prime}=0$, and the leading order of the slope is given by

$$
d v /\left.d x\right|_{+}=d v_{1} /\left.d \xi\right|_{\xi=\xi_{\varepsilon}} .
$$

Under the assumption (1.9), the second term in the bracket in the expression of $h$ in (4.7) is negligible; what we really need is

$$
\varepsilon^{1+\frac{n}{m-1}} q^{1+\frac{n}{m-1}} k^{2} \ll s .
$$

This assumption is consistent with (1.9). As $q=O(1)$ in the boundary layer, this corresponds to

$$
\varepsilon^{1+\frac{n}{m-1}} k^{2} / s=o(1) .
$$

However, this has to propagate to $x=x_{\varepsilon}$ where $q \sim(m-1) c \varepsilon^{-a}$, leading to the stronger condition

$$
\varepsilon^{\left(1+\frac{n}{m-1}\right)(1-a)} k^{2} / s=o(1),
$$

which is still compatible with (1.9).

Using (4.4) and (4.6), Equation (4.10) then leads to

$$
d v /\left.d x\right|_{+} \sim-s \varepsilon^{-\frac{n}{m-1}} e^{-A\left(\xi_{\varepsilon}\right)} \int_{-\infty}^{\xi} \frac{e^{A(\eta)}}{q^{1+\frac{n}{m-1}}(\eta)} d \eta, \quad A(\xi)=-\int^{\xi} \alpha(\beta) d \beta .
$$

Equation (4.4) and the definition of $\alpha$ in (4.8) yield, as $\xi \rightarrow+\infty$ :

$$
q(\xi) \sim(m-1) c \xi, \quad \alpha(\xi) \sim-\frac{1}{(m-1) \xi}, \quad e^{A(\xi)} \sim \xi^{\frac{1}{m-1}},
$$


and the lower bound of the integral of the first relation in (4.13) gives a bounded value. Then, according to (4.13), the expression of the leading order of the slope at the exit of the boundary layer is, for $\left(\xi-\xi_{\varepsilon}\right) / \xi_{\varepsilon}=o(1)$,

$$
\begin{gathered}
d v /\left.d x\right|_{+} \sim-\frac{s}{\mu \varepsilon^{\frac{n}{m-1}}} I(\xi), \quad \mu=[(m-1) c]^{1+\frac{n}{m-1}}, \\
I(\xi)=\frac{1}{\xi^{\frac{1}{m-1}}} \int^{\xi} \frac{1}{\eta^{1+\frac{n-1}{m-1}}} d \eta, \quad \begin{cases}n=1: & I(\xi)=\xi^{-\frac{1}{m-1}} \ln \xi, \\
n=0: & I(\xi)=m-1 .\end{cases}
\end{gathered}
$$

\subsection{Outer region.}

4.2.1. Equation. In this section, we investigate the hot region where the thermal length-scale is of the same order as the wavelength, $k x=O(1)$. We then introduce the outer coordinate,

$$
\zeta=k x
$$

The wavelength being larger than the thickness of the boundary layer, $k \ll 1 / \varepsilon$, the steady solution is a linear function of the coordinate in the outer region where $\zeta=O(1)$, $p \sim(m-1) c \zeta / k$. Introducing this expression into (1.7) leads to the following equation for $v=w(\zeta)$, expressed in term of the outer variable $\zeta$,

$$
-\zeta w^{\prime \prime}-\frac{w^{\prime}}{m-1}=\left(\frac{s}{\mu k^{1-\frac{n}{m-1}}} \frac{1}{\zeta^{\frac{n}{m-1}}}-\zeta\right) w, \quad v=w(\zeta), \quad w(+\infty)=0 .
$$

We have, in (4.18), thrown away the term $k^{-1} f^{\prime}(p) w$; a standard maximum principle argument shows that we may safely do this. That we can also throw away the terms containing $\varepsilon$ can be proved by an argment similar to Lemma 4.1 below; this will be omitted.

The downstream boundary condition is obtained as follows. Equation (4.18) is valid upstream to the cutoff $\lambda=\theta$ where $f=0$. This corresponds to

$$
\zeta<\zeta_{\theta}, \quad \zeta_{\theta}=\frac{k \theta}{(m-1) c} .
$$

According to (1.9), we are interested in the distinguished limit

$$
\varepsilon \rightarrow 0, \quad k \rightarrow \infty, \quad k \varepsilon \rightarrow 0 .
$$

In this limit the quantity $\zeta_{\theta}$ goes to infinity, $\zeta_{\theta} \rightarrow+\infty$. For large $\zeta$, Equation (4.18) reduces to $w^{\prime \prime} \sim w$, so that the boundary condition $v(+\infty)=0$ must hold since rejecting unbounded solutions yields $\lim _{\zeta \rightarrow+\infty} w=O\left(e^{-\zeta}\right)$. In physical terms this means that the disturbances vanish before reaching the region where the chemical heat is released.

4.2.2. Quasi-steady state approximation for $n=1$. Let us now anticipate that, for $n=1$, there is an outer solution which is in quasi-steady state approximation, meaning that the first term in the bracket of (4.18) is negligible, i.e.

$$
s k^{\frac{n}{m-1}-1} \zeta^{-\left(1+\frac{n}{m-1}\right)}=o(1) .
$$

As $\zeta=O(1)$ in the outer region, this means

$$
n=1: \quad s / k^{1-\frac{n}{m-1}}=o(1) .
$$


This corresponds to the fact that, due to the difference in density, the inertia term in the hot gases is negligible in front of the cold side one. Clearly this argument can no longer be used for $n=0$. The outer problem for $n=1$ is then reduced to solving

$$
n=1: \quad-\zeta w^{\prime \prime}-\frac{w^{\prime}}{m-1}+\zeta w=0, \quad w(+\infty)=0 .
$$

For $m=2$, the solution is the modified Bessel function $K_{0}(\zeta)$ which has a logarithmic divergence at $\zeta=0$. For $m>2$, the behavior of the solution near the origin is obtained by anticipating that $w$ approaches a constant value $v_{e}$, so that the last term in the left hand side of the first equation in (4.20) becomes negligible, yielding

$$
\left(\zeta^{\frac{1}{m-1}} w^{\prime}\right)^{\prime} \zeta^{1-\frac{1}{m-1}} \sim 0
$$

and thus

$$
n=1, m>2: \quad \lim _{\zeta \rightarrow 0^{+}}\left[\zeta^{\frac{1}{m-1}} w^{\prime}\right]=-\alpha_{m}, \quad\left(w-v_{e}\right) \sim-\frac{m-1}{m-2} \alpha_{m} \zeta^{\frac{m-2}{m-1}},
$$

where the value at the origin $v(0)=v_{e}$ is arbitrary and where $\alpha_{m}>0$ is a parameter of order unity depending on $m$, and obtained as an eigenvalue of (4.20) when the condition $w(0)=v_{e}$ is imposed. Notice that when $w$ in the last term in the left hand side of the first equation in (4.20) is replaced by $v_{e}$, integration of (4.20) between 0 and $\zeta$ yields

$$
\begin{gathered}
\xi^{\frac{1}{m-1}} w^{\prime}=-\alpha_{m}+\frac{m-1}{m} v_{e} \xi^{\frac{m}{m-1}}, \\
\left(w-v_{e}\right) \sim-\frac{m-1}{m-2} \alpha_{m} \zeta^{\frac{m-2}{m-1}}+\frac{m-1}{m} \frac{v_{e}}{2} \zeta^{2},
\end{gathered}
$$

which is the expansion of the solution to (4.20) around $\zeta=0$, pushed at the next order of (4.21). However, (4.20) is no longer valid in a tiny zone around the origin where the quasi-steady state fails, i.e. when $\zeta^{1+\frac{n}{m-1}}$ decreases sufficiently and becomes of the order of $s / k^{1-\frac{n}{m-1}}$. The outer solution cannot match the inner solution, and an intermediate layer must be introduced.

\subsection{Matching.}

4.3.1. Intermediate layer. Equation (4.18) may be re-written in the form

$$
-\left(\zeta^{\frac{1}{m-1}} w^{\prime}\right)^{\prime}=\left(\frac{s}{\mu k^{1-\frac{n}{m-1}}} \frac{1}{\zeta^{1+\frac{n}{m-1}-\frac{1}{m-1}}}-\zeta^{\frac{1}{m-1}}\right) w .
$$

The key point here is that we may neglect the variation of $w$. We state it in the

LEMMA 4.1. If $s / k^{1-\frac{n}{m-1}}=o_{k \rightarrow+\infty}(1)$, then in the area $\left\{\zeta^{1+\frac{n}{m-1}} \sim s / k^{1-\frac{n}{m-1}}\right\}$ we have $w=v_{e}\left(1+o_{k \rightarrow+\infty}(1)\right)$.

We postpone its proof until the end of the paragraph. Assume that it is true, $w \sim v_{e}$, Equation (4.24) may be integrated once to give for $\zeta>0$,

$$
\begin{aligned}
& n=1: \quad \zeta^{\frac{1}{m-1}} w^{\prime} \sim-\frac{s}{\mu k^{1-\frac{1}{m-1}}} v_{e} \ln \zeta-\alpha_{m}+\frac{m-1}{m} v_{e} \zeta^{\frac{m}{m-1}}, \\
& n=0: \quad \zeta^{\frac{1}{m-1}} w^{\prime} \sim-\alpha_{m}-\frac{s}{\mu k}(m-1) v_{e} \zeta^{\frac{1}{m-1}}+\frac{m-1}{m} v_{e} \zeta^{\frac{m}{m-1}}
\end{aligned}
$$


where the constant of integration $\alpha_{m}$ has been obtained by matching with the outer solution (4.21)-(4.22). Equation (4.25) is the expansion of the derivative of the solution to (4.18) for $\zeta>0$, around $\zeta=0^{+}$. Notice the difference between the two expressions, for $n=0$ the leading term is $\alpha_{m}$, while it is the logarithm for $n=1$.

4.3.2. The case $n=1$. For $n=1$, the non vanishing terms in the limit $\zeta \rightarrow 0^{+}$ of $k w^{\prime}$ in (4.25) match the expression (4.15)-(4.16) of $v_{x}^{\prime}$ in the limit $\xi \rightarrow+\infty$,

$$
n=1: \quad-\frac{s \ln \xi}{\mu \varepsilon^{\frac{1}{m-1}} \xi^{\frac{1}{m-1}}}=-\frac{k}{\zeta^{\frac{1}{m-1}}}\left(\frac{s \ln \zeta}{\mu k^{1-\frac{1}{m-1}}} v_{e}+\alpha_{m}\right), \quad \xi=\frac{x}{\varepsilon}, \quad \zeta=k x .
$$

The two $x^{-\frac{1}{m-1}} \ln x$ terms balance exactly for

$$
v_{e}=1
$$

and the identification of the $x^{-\frac{1}{m-1}}$ terms left lead to the relation $(s / \mu) \ln (k \varepsilon)=$ $-\alpha_{m} k^{1-\frac{1}{m-1}}$, which is the result in Theorem 1.2. Notice that the result is effectively consistent with the basic Assumption (4.19) and also with (4.12), leading to

$$
(k \varepsilon)^{\frac{m}{m-1}} \ln (1 / k \varepsilon)=o(1) .
$$

4.3.3. The case $n=0 . \quad$ For $n=0$, the expression in (4.25) with $\alpha_{m} \neq 0$ cannot match (4.15)-(4.16). Equation (4.19), (4.20), and (4.25) are no longer valid. Equation (4.18) for the outer region reads as

$$
-\zeta w^{\prime \prime}-\frac{w^{\prime}}{m-1}=\left(\frac{s}{(m-1) c k}-\zeta\right) w, \quad w(\zeta=+\infty)=0 .
$$

The result in Theorem 1.3, $s=c k$, is obtained by noticing that the corresponding outer solution $w=e^{-\zeta}$ matches directly the expressions in (4.15)-(4.16). It is also worth mentioning that the result is consistent with the initial Assumption (4.12).

\subsubsection{Proof of Lemma 4.1.}

Proof. It is enough to prove the case $n=1$. Choose $\zeta_{k} \ll 1$ and $\zeta_{k} \gg s / k^{1-\frac{n}{m-1}}$; this is allowed by the assumption $s / k^{1-\frac{n}{m-1}}=o_{k \rightarrow+\infty}(1)$. For $\zeta \geq \zeta_{k}$, Equation (4.24) has a positive zero-order coefficient; consequently it cannot attain its maximum on $\left[\zeta_{k},+\infty\left[\right.\right.$. Call $\hat{\zeta}_{k}$ the maximum of $v$ inside the interval $\left[k \varepsilon^{1-a}, \zeta_{k}\right]$, and normalise $v$ such that $v\left(k \varepsilon^{1-a}\right)=1$. On the interval $\left[\zeta_{\varepsilon}, 1\right]$ we may not assume the basic solution $p$ to be linear; however we may use Proposition 2.1 to obtain the following differential inequality for $v^{\prime}$, valid on $\left[\zeta_{\varepsilon}, 1\right]$ :

$$
\frac{d}{d \zeta}\left(\zeta^{\frac{1}{m-1}}\left(1+O\left(k^{\frac{2 m-1}{m-1}}\left(\varepsilon^{\frac{a}{m-1}}\right)\right) v^{\prime}\right)\right)=-(1+o(1))\left(\frac{s}{\mu k^{1-\frac{1}{m-1}} \zeta}-\zeta^{\frac{1}{m-1}}+\frac{d^{2} p}{d \zeta^{2}}\right) v
$$

hence

$$
\mid \frac{d}{\zeta}\left(\zeta^{\frac{1}{m-1}}\left(1+O\left(k^{\frac{2 m-1}{m-1}}\left(\varepsilon^{\frac{a}{m-1}}\right)\right) v^{\prime}\right) \mid \leq C\left(\frac{1}{\zeta}+\zeta^{\frac{1}{m-1}}+1\right) v \leq C\left(\frac{1}{\zeta}+1\right) v\left(\zeta_{\varepsilon}\right) .\right.
$$

Combining this inequality with the following one, obtained from the Harnack inequality,

$$
\left|v^{\prime}(1)\right| \leq C v\left(\zeta_{\varepsilon}\right),
$$


we obtain, by integrating from $\zeta_{\varepsilon}$ to 1 ,

$$
\forall \zeta \in\left[\zeta_{\varepsilon}, 1\right], \quad\left|v^{\prime}(\zeta)\right| \leq \frac{C v\left(\zeta_{\varepsilon}\right)}{\zeta^{\frac{1}{m-1}}}
$$

which in turn yields

$$
v(\zeta) \leq 1+C \zeta_{\varepsilon}^{\frac{m-1}{m-2}} v\left(\zeta_{\varepsilon}\right) .
$$

Because $\zeta_{\varepsilon} \leq \zeta_{k}=o(1)$ this bounds $v\left(\hat{\zeta}_{k}\right)$ as $v\left(\hat{\zeta}_{k}\right) \leq 1+o(1)$ and proves the lemma.

\section{Discussion of the results and perspectives}

We first discuss the mathematical implications of Theorem 1.1, and then we discuss its physical implications as well as the questions that it opens.

5.1. Relaxation to equilibrium. Let us explain what Theorem 1.1 says about relaxation to equilibrium in the initial model (1.4). Our point is that, due to the translation invariance in $x$, there is in general no reason why a solution to the Cauchy problem (1.4) should converge to a translate of $p(x)$ at the rate $e^{-s t}$, where $s$ is given by (1.10). What is true, however, is that the solution will become onedimensional (i.e. will converge to a $y$-independent function) at the rate $e^{-s t}$. In other words, it will lose its $2 \mathrm{D}$ character at this rate and, thus, can be expected to balance the strong (2D) hydrodynamical instability when the full model is considered. In order to keep the technical aspects as light as possible, we will show how the solution of (1.5) becomes 1D at the rate $e^{-s t}$. A nonlinear analysis could certainly be performed on problem (1.4), but it would only be much more technical and we therefore omit it from this paper.

Write the linearised problem (1.5) under the abstract form

$$
\left\{\begin{aligned}
u_{t}-a(x) \Delta u+b(x) u_{x}+c(x) u & =0, & & \left(t>0,(x, y) \in \mathbb{R}^{2}\right), \\
u(t, \pm \infty, y) & =0, & & u\left(t, x, y+\frac{2 \pi}{y}\right)=u(t, x, y) .
\end{aligned}\right.
$$

Look - this is once again a standard idea in the study of elliptic operators whose coefficients are one-dimensional - for $u(t, x, y)$ with the form

$$
u(t, x, y)=e^{\alpha(x)} v(t, x, y), \quad \alpha^{\prime}(x)=\frac{b(x)}{2 a(x)} .
$$

Of course, we have here $0<a_{-} \leq a(x) \leq a_{+}<+\infty$. Then the new unknown $v(t, x, y)$ satisfies

$$
\left\{\begin{aligned}
v_{t}-a(x) \Delta v+d(x) u & =0, & & \left(t>0,(x, y) \in \mathbb{R}^{2}\right), \\
v(t, \pm \infty, y) & =0, & & v\left(t, x, y+\frac{2 \pi}{y}\right)=v(t, x, y),
\end{aligned}\right.
$$

where we have $d(x)=c(x)-\frac{b^{2}(x)-a^{\prime}(x) b(x)-b^{\prime}(x) a(x)}{2 a(x)}$. For all $n \in \mathbb{Z}$, let $L^{n}$ be the one-dimensional operator $L^{n}=-a(x) \frac{d^{2}}{d x^{2}}+d(x)+n k^{2} a(x)$, and let $s_{n}(k)$ be the eigenvalue corresponding to the $\lambda_{0}$ given by Theorem 3.1. We have, with the notation of Theorem 3.1, $c(x)=\frac{1}{a(x)}$. Expand $v(t, x, y)$ in its Fourier series as

$$
v(t, x, y)=\sum_{n \in \mathbb{Z}} e^{i n k y} v^{n}(t, x)
$$


where $v^{n}$ solves

$$
\left(\partial_{t}+L^{n}\right) v^{n}=0, \quad(t>0, x \in \mathbb{R}) .
$$

We assume (consistent with (1.5)) that $L^{0}$ has a positive zero eigenfunction. From the maximum principle, the sequence $\left(s_{n}(k)\right)_{n}$ is increasing in $n$; moreover we have, for $n \neq 0$,

$$
\left\|v_{n}(t, .)\right\|_{L^{2}(\mathbb{R})} \leq e^{-s_{n}(k) t}\left\|v_{n}(0, .)\right\|_{L^{2}(\mathbb{R})} \leq e^{-s_{1}(k) t}\left\|v_{n}(0, .)\right\|_{L^{2}(\mathbb{R})} .
$$

By Parseval's identity we have

$$
\left\|v(t, . . .)-v^{0}(t, .)\right\|_{L^{2}(\mathbb{R} \times(0,1))} \leq e^{-s_{1}(k) t}\|v(0, ., .)\|_{L^{2}(\mathbb{R} \times(0,1))} .
$$

The same estimate would hold for $\left\|v_{y}(t, . . .)\right\|_{L^{2}(\mathbb{R} \times(0,1))}$, just by differentiating (1.5) with respect to $y$. Now, in our application we have

$$
s_{1}(k)=s, \quad \alpha^{\prime}(x)=\frac{1}{p}\left(1-\frac{2}{m-1} p^{\prime}\right) \sim_{x \rightarrow-\infty} \frac{1}{\varepsilon} .
$$

And estimate (5.3) indeed yields the convergence rate $s$ to the 1D component of $v$.

5.2. Physical discussion. The relaxation problem under investigation presents two specificities. First, the diffusion length-scale increases strongly across the reaction-diffusion wave. Secondly, the attention is focused to disturbances whose wave length lies in an intermediate range, smaller than the largest diffusion lengthscale and larger than the smallest one.

The motivation of the analysis was to investigate the diffusive relaxation mechanism counterbalancing at small wave lengths very strong hydrodynamical instabilities, such as the Rayleigh-Taylor instability with a small Froude number (based on the total thickness of the wave). The main outcome is that Theorem 1.1 is close to the approximate result obtained when including all the hydrodynamical effects; see [10] and the references herein. The difference comes from the logarithmic term. Is this logarithmic term still significant when the hydrodynamics are fully taken into account? This is an open question that will be investigated in the near future by an extension of the present work.

It is also worth recalling that the Theorem 1.3, for $n=0$ and $m>2$, is quite different from the relaxation of a reaction-diffusion wave with a single diffusion lengthscale $m=0$. In the latter case one gets $s=k^{2}$ for every $k$.

Acknowledgement. The three authors were supported by the CEA program 'Instabilités hydrodynamiques en FCI'.

\section{REFERENCES}

[1] H. Berestycki and B. Larrouturou, Quelques aspects mathmatiques de la propagation des flammes prmlanges, Nonlinear Partial Differental Equations and Applications, Sminaires du Collge de France, X, Brzis et Lions diteurs, Pitman, Londres, 65-129, 1991.

[2] H. Berestycki and L. Rossi, On the principal eigenvalue of elliptic operators in $\mathbb{R}^{N}$ and applications, J. Eur. Math. Soc. (JEMS), 8, 195-215, 2006.

[3] L.A. Caffarelli and A. Friedman, Regularity of the free boundary for the one-dimensional flow of gas in a porous medium, Amer. J. Math., 101, 1193-1218, 1979.

[4] L.A. Caffarelli and N. Wolanski, $C^{1, \alpha}$ regularity of the free boundary for the $N$-dimensional porous media equation, Commun. Pure Appl. Math., 43, 885-902, 1990. 
[5] P. Clavin, Dynamics of combustion fronts in premixed gases: from flames to detonations, Proceedings of the Combustion Institute, 28, 569-585, 2000.

[6] P. Clavin and L. Masse, Instabilities of ablation fronts in inertial confinement fusion, Phys. Plasmas, 11, 690-705, 2004.

[7] L. Glangetas and J.M. Roquejoffre, Bifurcation of travelling waves in the thermo-diffusive model for flame propagation, Arch. Rat. Mech. Anal., 134, 341-402, 1996.

[8] B. Helffer and O. Lafitte, The semiclassical regime for ablation front models, Arch. Ration. Mech. Anal., 183, 371-409, 2007.

[9] M. Reed and B. Simon, Methods of Modern Mathematical Physics, Vol. II, New York, Academic Press.

[10] J. Sanz, L. Masse and P. Clavin, The linear Darrieus-Landau and Rayleigh-Taylor instabilities in inertial confinement fusion revisited, Phys. Plasmas, 13, 102702, 2006. 